\title{
Density functional theory of freezing for hexagonal symmetry: Comparison with Landau theory
}

\author{
Brian B. Laird, John D. McCoy, and A. D. J. Haymet \\ Department of Chemistry, University of California, Berkeley, California 94720
}

(Received 7 August 1987; accepted 10 December 1987)

\begin{abstract}
Density functional theory, studied recently by us [J. Chem. Phys. 87, 5449 (1987)] is used to study the freezing of hard disks and hard spheres into crystals with hexagonal symmetry. Two different numerical techniques are used, namely a Gaussian approximation to the crystal density and a more general Fourier expansion of the crystal density. The results from these methods are compared with each other, more approximate versions of density functional theory, and computer simulations. In addition, we compare density functional theory with Landau theories of first order transitions, in which the free energy is expanded as a power series, usually in just one order parameter. We find that traditional Landau theory has little validity when applied to the freezing transition.
\end{abstract}

\section{INTRODUCTION}

Classical density functional methods ${ }^{1-6}$ have described successfully the freezing of monatomic, spherically symmetric systems, ${ }^{7-9}$ and more recently the freezing of mixtures, ${ }^{10,11}$ molecular liquids which have orientational degrees of freedom, ${ }^{12,13}$ and even water. ${ }^{14}$

In a previous paper, ${ }^{8}$ hereafter denoted paper I, the present authors examined in detail two computational techniques for implementing the density functional theory of freezing. One method assumes that the crystal phase density can be approximated by Gaussians. The other, more general method expands the crystal density as a sum of Fourier components which are allowed to vary independently, subject only to the symmetry constraints of the crystal lattice under investigation. In paper I we corrected inconsistencies in earlier versions of the Fourier expansion method, and examined carefully the freezing of the hard sphere and Lennard-Jones liquids into face-centered-cubic ( $\mathrm{fcc}$ ) crystals using both the Gaussian approximation and the corrected Fourier expansion method. Both methods predict similar thermodynamic properties for the freezing transition, but the Fourier method results exhibit significant crystal density anharmonicities and anisotropies which are excluded $a$ priori in the Gaussian approximation.

In this paper we compare the density functional theory of freezing with Landau theory, which is extremely important in the theory of second order phase transitions, but which turns out not to be a useful theory of first order transitions such as freezing. In addition, we extend the analysis of Paper I to the freezing of hard particles into structures with hexagonal symmetry, in both two dimensions $(d=2)$ to the triangular lattice, and in three dimensions $(d=3)$ to the hexagonally close-packed (hcp) lattice. The freezing of hard spheres into the hcp structure has been examined previously, by Yussouff ${ }^{15}$ and by Igloi ${ }^{16}$ using the Fourier method, and by Baus and $\mathrm{Colot}^{17}$ using a variant of the Gaussian approximation. However, according to paper I, both Fourier method studies used an inconsistent version of the Fourier expansion, and too few order parameters. In addition, Yussouff ${ }^{15}$ used an unnecessarily complicated and, from our analysis, incorrect representation of the hcp order parameters. Ramakrishnan, ${ }^{18}$ Tarazona and co-workers ${ }^{19,20}$ and Colot and Baus $^{21}$ have all examined the freezing of hard disks using approximate versions of the density functional theory, and we compare our predictions with their calculations and earlier computer simulations. ${ }^{22,23}$

In Sec. II we summarize the freezing formalism for both Gaussian and Fourier methods. In Sec. III we use simple mathematical approximations to derive Landau theory from density functional theory, and use the Landau free energy to try to predict the relative stability of crystal phases. Landau expansions have become quite popular recently for studies of first order transitions, and a comment on their inconsistencies and pathologies is in order. In Secs. IV and V we collect our predictions for two and three dimensional hard particles, and compare them with other methods, and collect our conclusions in Sec. VI. Since the hep Fourier freezing formalism is more difficult to implement, and seems to have confused some workers, we describe complete technical details in the Appendix.

\section{DENSITY FUNCTIONAL THEORY OF FREEZING}

Consider a system of classical particles with temperature $T$, volume $V$, chemical potential $\mu$, and interaction potential $U\left(\mathbf{r}_{1}, \mathbf{r}_{2}, \ldots\right)$. The goal of density functional theory is to construct a free energy functional from which the structural and thermodynamic properties of a spatially inhomogeneous phase (in this case, the crystal) can be determined. This could be accomplished in a variety of ways. Following paper I, we choose to expand the functional about a well characterized reference state, in this case the equilibrium uniform liquid, using thermodynamic perturbation theory. The details of how to do this in the grand canonical ensemble are given in paper I, and are recapitulated here.

The equilibrium density $\rho(\mathbf{r})$ of the inhomogeneous (crystal) phase is the density which minimizes the functional 


$$
\begin{aligned}
& \Delta \beta \tilde{\omega}[\rho(\mathbf{r})] \\
&=\frac{1}{\rho_{L} V} \int d \mathbf{r}_{1}\left\{\rho\left(\mathbf{r}_{1}\right) \ln \left[\rho\left(\mathbf{r}_{1}\right) / \rho_{L}\right]\right. \\
&\left.-\left[\rho\left(\mathbf{r}_{1}\right)-\rho_{L}\right]\right\} \\
&-\frac{1}{2 \rho_{L} V} \int d \mathbf{r}_{1} d \mathbf{r}_{2} c\left(\left|\mathbf{r}_{1}-\mathbf{r}_{2}\right|\right)\left[\rho\left(\mathbf{r}_{1}\right)-\rho_{L}\right] \\
& \times\left[\rho\left(\mathbf{r}_{2}\right)-\rho_{L}\right],
\end{aligned}
$$

where $\beta^{-1}=k T, \rho_{L}$ is the density of the coexisting, uniform bulk liquid phase, and $c(r)$ is the Ornstein-Zernike direct correlation function of the bulk liquid. Note that the dependence of the theory on the interaction potential $U$ is contained implicitly in $c(r)$, and there is not necessarily a restriction to pairwise additive forces. The value of the functional $\Delta \beta \widetilde{\omega}$ at the minimizing density $\rho(\mathbf{r})$ is

$$
\Delta \beta \omega=\frac{\beta \Omega_{\text {crystal }}-\beta \Omega_{\text {liquid }}}{N_{L}}=\min _{\rho(\mathbf{r})} \Delta \beta \widetilde{\omega}[\rho(\mathbf{r})],
$$

where $\Omega$ is the grand thermodynamic potential and $N_{L}$ is the average number of liquid particles.

The central approximation of the theory is that the correlation functions of the crystal are expanded as functional Taylor series about the reference liquid, and truncated after second order. The effect of these approximations is discussed in paper I and by Haymet and Oxtoby. ${ }^{24}$ Various versions of density functional theory differ principally in the choice of reference liquid. Our choice avoids consideration of hypothetical liquids with densities intermediate between equilibrium liquid and crystal, and also extra terms involving the chemical potential difference. To a given order in perturbation theory, the equilibrium freezing transition is located when the pressures (as well as the temperatures and chemical potentials) of the crystal and liquid are equal, that is, $\Delta \beta \omega=0$.

To date, the functional (2.1) has been minimized by two different methods. In one method, the crystal density is assumed to be Gaussian and expanded in the form,

$$
\rho(\mathbf{r})=\left(\pi \epsilon^{2}\right)^{-3 / 2} \sum_{\left\{\mathbf{R}_{n}\right.} \exp \left[-\left|\mathbf{R}_{n}-\mathbf{r}\right|^{2} / \epsilon^{2}\right],
$$

where the vectors $\left\{\mathbf{R}_{n}\right\}$ are the real space crystal lattice vectors and $\epsilon$ is a measure of the width of the Gaussian peaks. If the Gaussian peaks are assumed to be nonoverlapping, this choice of density ${ }^{25}$ leads to a simple evaluation of the $\rho \ln \rho$ term in Eq. (2.1). The integral over $c(r)$, which is the $\rho c(r) \rho$ term in Eq. (2.1), is evaluated most easily by converting the real space integration into a Fourier sum over reciprocal lattice vectors (RLVs) $\left\{\mathbf{k}_{n}\right\}$. The Fourier transform of the direct correlation function $c(k)$ is related to the experimentally measured structure factor $S(k)$ via,

$$
c(|\mathbf{k}|)=\rho \int d \mathbf{r} e^{i \mathbf{k} \cdot \mathbf{r}} c(r)=1-\frac{1}{S(k)} .
$$

The functional (2.1) for the special case of Gaussian crystal density may then be written

$$
\begin{aligned}
\Delta \beta \omega= & 1-(1+\eta)\left[\frac{5}{2}+\ln \rho_{L}+\frac{3}{2} \ln \left(\pi \epsilon^{2}\right)\right] \\
& -\frac{1}{2} \eta^{2} c(0)-\frac{1}{2} \sum_{\left(\mathbf{k}_{n}\right.} c\left(\left|\mathbf{k}_{n}\right|\right) \mu^{2}\left(\left|\mathbf{k}_{n}\right|\right) \\
& -\frac{1}{6} \eta^{3} c^{(3)}(0,0),
\end{aligned}
$$

where $\eta$ is the fractional density change on freezing

$$
\eta=\left(\rho_{S}-\rho_{L}\right) / \rho_{L},
$$

and $\rho_{L} \mu_{n}\left(\mathbf{k}_{n}\right)$ is the Fourier component of the crystal density corresponding to the (nonzero) $\operatorname{RLV~} \mathbf{k}_{n}$,

$$
\rho(\mathbf{r})=\rho_{L}\left[1+\eta+\sum_{\left\{\mathbf{k}_{n}\right\}} \mu\left(\mathbf{k}_{n}\right) e^{i \mathbf{k}_{n} \cdot \mathbf{r}}\right] .
$$

For the Gaussian density (2.3), this component is

$$
\mu_{G}\left(\mathbf{k}_{n}\right)=(1+\eta) \exp \left(-\left|\mathbf{k}_{n}\right|^{2} \epsilon^{2} / 4\right) .
$$

The last term in Eq. (2.5) is the zero wave vector contribution to the third order term in the perturbation expansion of $\Delta \beta \tilde{\omega}$, not explicitly shown in Eq. (2.1). This term involves the three particle direct correlation $C^{3}\left(\mathbf{r}_{1}, \mathbf{r}_{2}, \mathbf{r}_{3}\right)$, of which the zero wavevector component is given by

$$
\begin{aligned}
c^{3}(0,0) & =\rho_{L}^{2} V^{-1} \int_{V} d \mathbf{r}_{1} d \mathbf{r}_{2} d \mathbf{r}_{3} C^{3}\left(\mathbf{r}_{1}, \mathbf{r}_{2}, \mathbf{r}_{3}\right) \\
& =\rho_{L} \frac{\partial c(0)}{\partial \rho_{L}}-c(0) .
\end{aligned}
$$

The reasons for including this term are discussed in paper $I$.

At a fixed liquid density and in the Gaussian approximation, Eq. (2.5) is a function of two parameters only, the Gaussian width $\epsilon$ and the fractional density change $\eta$. Minimization with respect to these two quantities is straightforward.

The second method uses the Fourier expansion (2.7) but allows each nonsymmetry related Fourier component to vary independently. This greater generality enables this method to seek possible anharmonicities and anisotropies in the crystal density. As discussed in paper $I$, in the absence of information about the correlation functions for point defects such as interstitials and vacancies, it is reasonable to assume that the crystal (of whatever symmetry) is "perfect" in the sense that there is exactly one particle per lattice site,

$$
\int_{\Delta} d \mathbf{r}_{1} \rho\left(\mathbf{r}_{1}\right)-N_{\Delta}=0,
$$

where $N_{\Delta}$ is the number of atoms per unit cell of volume $\Delta$.

Minimizing Eq. (2.1) subject to the constraint (2.10) yields, as shown in paper $I$,

$$
\ln \left[\rho\left(\mathbf{r}_{1}\right) / \rho_{L}\right]=\lambda+\int_{V} d \mathbf{r}_{2} c\left(\left|\mathbf{r}_{1}-\mathbf{r}_{2}\right|\right)\left[\rho\left(\mathbf{r}_{2}\right)-\rho_{L}\right],
$$

where $\lambda$ is the Lagrange multiplier which enforces the constraint (2.10). Inserting the Fourier decomposition (2.7), we obtain a set of coupled nonlinear equations for the order parameters and the Lagrange multiplier,

$$
\begin{aligned}
\mu\left(\mathbf{k}_{j}\right)+\delta_{\mathbf{k}_{j, 0}}= & \int \frac{d \mathbf{r}}{\Delta} e^{-i \mathbf{k}_{j} * \mathbf{r}} \exp \{\lambda+c(0) \eta \\
& +\frac{1}{2} c^{(3)}(0,0) \eta^{2} \\
& \left.+\sum_{\left\{\mathbf{k}_{n}\right.} c\left(\left|\mathbf{k}_{n}\right|\right) \mu\left(\mathbf{k}_{n}\right) e^{\lambda \mathbf{k}_{n} \cdot \boldsymbol{r}}\right\} .
\end{aligned}
$$

Equation (2.12) is solved together with the constraint equation

$$
\rho_{L}(1+\eta)=N_{\Delta} / \Delta
$$


to yield the crystal density. In principle, the sum in the integrand of Eq. (2.12) is over an infinite set of RLVs, but this sum is eventually self-truncating because the coefficients $c\left(\mathbf{k}_{n}\right)$ tend to zero for large amplitude wave vectors. Misleading results are obtained if too few vectors are retained in this sum. ${ }^{8}$

At the minimum value, the value of the functional is the grand thermodynamic potential difference,

$$
\begin{aligned}
\Delta \beta \widetilde{\omega}_{\min }= & {[c(0)-1] \eta+\frac{1}{2}\left[c(0)+c^{(3)}(0,0)\right] \eta^{2} } \\
& +\frac{1}{3} c^{3}(0,0) \eta^{3} \\
& +\frac{1}{2} \sum_{\left\{\mathbf{k}_{n}\right\}} c\left(\left|\mathbf{k}_{n}\right|\right) \mu^{2}\left(\mathbf{k}_{n}\right)+\lambda(1+\eta) .
\end{aligned}
$$

In this form $\Delta \beta \widetilde{\omega}_{\min }$ is minimized with respect to the cell volume $\Delta$, to give the perfect crystal minimum of the functional. Using either method, the value of the liquid density is varied until the difference in grand potential between the crystal and liquid is zero. This means that the pressures of the two phases are equal, and since the temperatures and chemical potentials of the two phases are equal by construction, this identifies the freezing point. These theories are applied to hard particles in two and three dimensions in Secs. IV and V below.

\section{COMPARISON WITH LANDAU THEORY}

The simplest theories of phase transitions are those based on the free energy expansion introduced by Landau. ${ }^{26}$ Within this approximation, a set of order parameters $\left\{\phi_{n}\right\}$ are chosen and the free energy is expanded in a polynomial series about the transition point,

$F=\sum_{m=1}^{\infty} \sum_{a_{1}+a_{2}} \sum_{+a_{n}=m} A_{m}\left(a_{1}, \ldots, a_{n}\right) \phi_{1}^{a_{1}} \phi_{2}^{a_{2}} \ldots \phi_{n}^{a_{n}}$,

where $F$ is the free energy relative to the disordered phase in which the order parameters vanish. Equation (3.1) is then truncated at low order, typically $m=4$ or $m=6$, and the physics determined by minimizing the resulting expression for the free energy.

These theories have been extremely successful for second order transitions, both in meanfield theory and as the starting point for renormalization group analysis, because often the higher order terms in the expansion (3.1) can be shown to be "irrelevant" in the formal sense. . $^{27,28}$

It is a much more risky proposition to use this expansion for first order transitions such as freezing. Often the choice of order parameters deemed to be relevant (in any sense) is motivated by convenience rather than physical reality. Usually the density change on freezing is completely ignored, although it often dominates the physics of first order phase transitions. Furthermore, we show here that the higher order terms in the expansion (3.1) are just as large as, say the $m=4$ term, and omitting them leads to qualitatively incorrect physics.

In an important paper, Alexander and McTague ${ }^{29}$ used a Landau free energy, with the fourth order term approximated by the square of the second order term, to study the freezing transition. They concluded that for systems which have small density changes on freezing, and small latent heats, freezing to a bcc phase is favored over fcc. Their argument, which we claim is incorrect, both quantitatively and qualitatively, may be summarized as follows. Let the free energy of the inhomogeneous phase relative to the bulk liquid be given by

$$
F=F_{2}+F_{3}+F_{4}+\cdots,
$$

where $F_{n}$ is the $n$th order term in the Landau expansion, and the order parameters are the Fourier components of the crystal density, just as in Sec. II above. The quadratic term has the form

$$
F_{2}=\sum_{\mathbf{k}} A_{2}(\mathbf{k}) \rho_{\mathrm{k}} \rho_{-\mathbf{k}},
$$

where $A(k)$ depends only on the magnitude of the wave vector since the liquid is isotropic. Alexander and McTague argue that as the transition is approached, $A_{2}(\mathrm{k})$ has a minimum at some wave vector $|\mathbf{k}|=q$, and that at the freezing point it becomes favorable to form a crystal with reciprocal lattice vectors of this magnitude. Neglecting all other Fourier components, they assume that the order parameter

$$
\rho_{q}^{2}=\sum_{|\mathbf{k}|=q} \rho_{\mathbf{k}}^{2}
$$

dominates the transition, and proceed to evaluate the possible third order terms which can be constructed from this order parameter. The third order term has the form

$F_{3}=A_{3}(q) \sum_{\left|\mathbf{k}_{1}\right|=q\left|\mathbf{k}_{2}\right|=q} \sum_{\left|\mathbf{k}_{3}\right|=q} \delta\left(\mathbf{k}_{1}+\mathbf{k}_{2}+\mathbf{k}_{3}\right) \rho_{\mathbf{k}_{1}} \rho_{\mathbf{k}_{2}} \rho_{\mathbf{k}_{3}}$,

where the $\delta$ function constrains the three RLVs to form an equilateral triangle of side length $q$, as required by the invariance properties of $F_{3}$. Thus, if one identifies $q$ as the amplitude of the nearest neighbor RLVs in a crystal, only certain lattice symmetries (such as bcc, but not fcc) have nonzero third order contributions to the free energy.

The simplest such symmetry is six vectors which form a hexagon. This is the first RLV set for the $d=2$ triangular lattice as well as for $d=3$ hexagonal lattices such as hcp. The next such symmetry is 12 vectors forming an octahedron, which is the first RLV set of the bcc real space structure. The only other case is 30 vectors forming an icosahedron, which of course is not a subset of any periodic lattice but which has obvious appeal for the study of icosahedral quasicrystals..$^{30,31}$ Simple geometric analysis shows that the third order coefficient is most negative for the octahedron case, leading Alexander and McTague to conclude that the boc structure is favored uniquely by the third order term.

Inspired by Alexander and McTague, ${ }^{29}$ a number of authors have used a similar analysis to study the stability of quasicrystals in single ${ }^{30}$ and multicomponent ${ }^{31}$ systems. Klein and Leyvraz ${ }^{32}$ have studied nucleation near the meanfield spinodal line, and Shih et $a l^{33}$ have constructed a Landau theory of the bcc crystal-liquid interface, studied earlier with density functional theory by Oxtoby and Haymet. ${ }^{34,35}$ Despite the many approximations detailed below, some authors have tried to claim a generality of results from Landau theory, similar to second order phase transitions. We show explicitly that this is incorrect.

We attempt to summarize here the problems with the traditional Landau analysis of first order transitions. Alexander and McTague want their analysis to be valid for transitions where the first-order character is "not too pronounced". This is 
the only possible justification for neglecting the zero-wavevector-order parameter, the fractional density change on freezing, yet even this is incorrect. Even for alkali metals this term makes a large contribution to the properties of the transition. In the incompressible limit, ${ }^{36}$ the fractional density change does vanish but the zero-wave-vector coefficient $c(0)$ tends to negative infinity in such a way that the product $\eta c(0)$ is finite, and this term contributes to the free energy.

The Landau analysis would also predict that the hep structure is much more stable than the fcc structure, since its RLVs contribute to a third order term. This is obviously false, since for simple substances such as the inert elements, the fcc and hcp structure are known to be separated by tiny free energy differences. For hard spheres there is no significant difference in free energy according to full density functional theory, as shown in Sec. V.

The appeal of the Alexander-McTague approach is that it reduces the seemingly unmanageable dependence of the free energy upon all the Fourier components of the single-particle density to a dependence upon a single component. Indeed, when the freezing transition is investigated experimentally, only a single Fourier component of the density $\rho_{K}$ need be observed in order to locate the transition point. From the standpoint of density functional theory, it is possible to reduce the free energy functional (2.1) to a function of any given $\rho_{K}$ by partial minimization with respect to all other Fourier components. However, because the other Fourier components play a great role in determining the form of this single parameter free energy, the shape of the resultant functional cannot be predicted on the basis of the symmetry of the given $\rho_{K}$ alone, as is assumed by Alexander and McTague. It is this assumption (unjustified, in our opinion) which is removed by density functional theory. The reciprocal lattice is not made of just nearest neighbor RLVs, but an interlocking network of RLVs which obey the symmetry constraints of the crystal. Some Fourier components of the crystal density lower the free energy with respect to the uniform liquid, but others raise it, and the actual transition represents a delicate balance between these competing effects. In other words, some Fourier components which are necessary to form the crystal actually cost free energy, but this is compensated by other Fourier components which lower the free energy. Usually the zero wave vector component lowers the free energy, but in some substances such as water ${ }^{14}$ which expand on freezing, it disfavors the crystal, and the network of nonzero vectors must compensate for this.

More explicitly, one can calculate from first principles the coefficients in the Landau expansion, and show that the coefficients in the quadratic term are comparable for many wave vectors. Expanding the crystal density as

$$
\rho(\mathbf{r})=\rho_{L}(1+\eta)\left[1+\sum_{\mathbf{k}}^{\prime} \phi_{\mathbf{k}} e^{i \mathbf{k} \cdot \mathbf{r}}\right],
$$

where the prime indicates that only nonzero vectors are included in the sum, we may expand the functional [Eq. (2.1)] to quadratic order to obtain

$$
\begin{aligned}
\Delta \beta \widetilde{\omega}= & \frac{1}{2}[1-c(0)] \eta^{2} \\
& +\frac{1}{2} \sum_{i}^{\prime} n_{i}\left[1-c\left(\left|\mathbf{k}_{i}\right|\right)\right] \phi_{i}^{2}+\cdots,
\end{aligned}
$$

where $i$ labels a set of symmetry related RLVs and $n_{i}$ is the number of elements in RLV set $i$. Notice that the Fourier expansion (3.6) differs from expansion (2.7) in that the order parameters are scaled by the solid density rather than the liquid density. This change greatly simplifies the Landau expansion of Eq. (2.1). For hard spheres near the freezing point $\left(\rho_{L}\right.$ $=0.95$ ), the quadratic coefficients for the first five RLV sets of a hypothetical bcc hard sphere crystal are

$$
\begin{aligned}
& A_{2}(000)=\frac{1}{2}[1-c(0)]=31, \\
& A_{2}(110)=6\left[1-c\left(k_{1}\right)\right]=2.46, \\
& A_{2}(200)=3\left[1-c\left(k_{2}\right)\right]=5.01, \\
& A_{2}(211)=12\left[1-c\left(k_{3}\right)\right]=8.52, \\
& A_{2}(220)=6\left[1-c\left(k_{4}\right)\right]=6.05,
\end{aligned}
$$

where $A_{2}(\mathrm{hkl})$ is the quadratic coefficient corresponding to the RLV set containing the RLV (hkl).

In fact, Landau theory with just one order parameter is unable to predict freezing at all! Expanding the functional (2.1) in terms of the fractional density change $\eta$ and the order parameter for the first nonzero RLV $\phi_{1}$ gives

$$
\begin{aligned}
\Delta \beta \widetilde{\omega}(\eta, \phi)= & \frac{1}{2}[1-c(0)] \eta^{2}-\eta+(1+\eta) \ln (1+\eta) \\
& -\frac{1}{2}(1+\eta)^{2} c\left(k_{1}\right) f(2) \phi_{1}^{2} \\
& +(1+\eta) \sum_{n=2}^{\infty} \frac{(-1)^{n} f(n)}{n^{2}-n} \phi_{1}^{n},
\end{aligned}
$$

where the function $f(n)$ is defined as the number of different $n$ gons which can be formed from members of the first RLV set. The values of $f(n)$ for $n$ equals two to six are displayed in Table I for both the bcc $\left(\phi_{1}=\phi_{110}\right)$ and fcc $\left(\phi_{1}=\phi_{111}\right)$ cases. The terms involving $\eta$ can be further expanded as

$$
-\eta+(1+\eta) \ln (1+\eta)=\sum_{n=2}^{\infty} \frac{(-1)^{n}}{n^{2}-n} \eta^{n} .
$$

For the two-order parameter theory, the bcc expansion (fourth order in both order parameters) yields only the liquid minimum unless the coefficient $c\left(k_{1}\right)$ is allowed to assume a completely unphysical value $c\left(k_{1}\right)>14 / 15=0.933$, which corresponds to a liquid structure factor $S\left(k_{1}\right)>15$. At this unphysical minimum the fractional density change vanishes, $\eta=0$. There is no change in this result even if the $\phi_{110}$ expansion is taken to sixth order and the $\eta$ expansion is taken to infinite order. For the fcc expansion there is only the liquid minimum, for any values of the coefficients $c(0)$ and $c\left(k_{1}\right)$, and this remains true even if all higher order terms in both order parameters are included.

We conclude that analysis of freezing transition must include all relevant order parameters. Fortunately there is at least one way to do this, as shown in Sec. II, and there is absolutely no need to apply unnecessary mathematical approximations, such as expanding the logarithms in Eq. (2.1) to obtain a power series, when the full functional is tractable. We note further that some analyses which appeal to Landau theory confuse the grand functional (2.1) with the canonical (Helmholtz) free energy which always contains linear terms in $\eta$. There is little point in correcting these problems since theories which rely on polynomial expansions have little validity, even qualitatively. 


\section{FREEZING OF HARD DISKS}

We have applied the Gaussian and Fourier space methods of freezing to the hard disk system despite the absence of a "true" two-dimensional crystal. The equations used by the two methods in two dimensions are essentially the same as used in three dimensions and will not be reproduced here. In this paper, we are concerned only with hard particles, hard disks in this section and hard spheres in the next. The interaction potential for these systems is given by

$$
u(r)=\left\{\begin{array}{ll}
\infty, & r<1 \\
0, & r \geqslant 1
\end{array},\right.
$$

where the particle diameter $\sigma$ has been used as the length scale both here and through out this paper. The liquid direct correlation function, which is the principal input to the calculation, has been obtained both from an ansatz developed by Leutheusser $^{37}$ and from an ansatz developed by Colot and Baus. ${ }^{21}$ Both ansatzes are developed to fit solutions of the OrnsteinZernike equation with a Percus-Yevick closure. Figure $1 \mathrm{com}-$ pares the deviations of the two approximate expressions, evaluated at $k=0$, from the exact solution as given by $\mathrm{Lado} .{ }^{38} \mathrm{It}$ is worth restating that in even dimensions the Percus-Yevick equation of hard particles has not been solved analytically. While both ansatzes behave well at low to moderate densities, the Colot-Baus $c(k)$ is superior at solidlike densities.

In two dimensions $(d=2)$, we considered two lattice types: the square and the triangular. For the square lattice, the unit real space vectors are

$$
\mathbf{a}_{1}=d \mathbf{i}
$$

and

$$
\mathbf{a}_{2}=d \mathbf{j}
$$

and, for the triangular case:

$$
\mathbf{a}_{1}=d \mathbf{i}
$$

and

$$
\mathbf{a}_{2}=-(d / 2) \mathbf{i}+(\sqrt{3} d / 2) \mathbf{j},
$$

where $i$ and $j$ are the Cartesian unit vectors in the orthogonal $x$ and $y$ directions, respectively. The average nearest neighbor separation is given by $d$. A crystal is said to be perfect if the

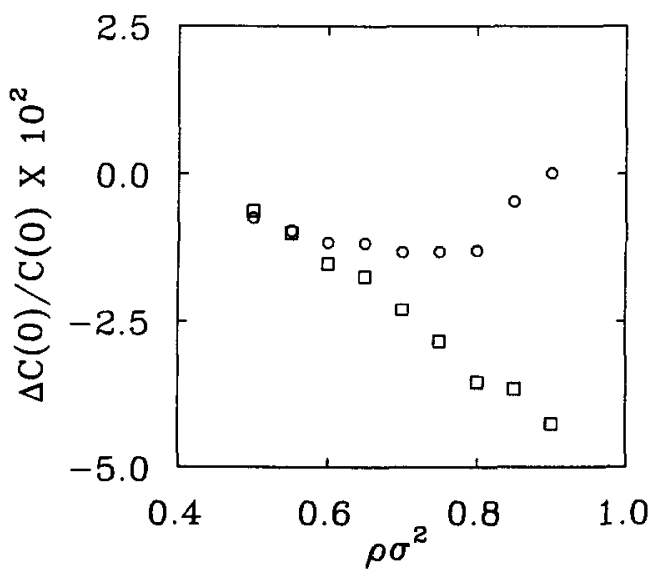

FIG. 1. The deviation of the zero wave vector hard disk direct correlation function $c(k=0)$ for the ansatz of Colot and Baus (Ref. 17) (circles) and for the ansatz of Leutheusser (Ref. 37) (squares) from the exact (numerical) solution of the Percus-Yevick equation by Lado (Ref. 38), as a function of liquid density. density is constrained so that the number of particles is equal to the number of particle sites; otherwise, the crystal is said to be "imperfect". The degree to which a crystal is imperfect can be indicated by the quantity $A$ which is defined as the number of particles divided by the number of particle sites. The Sutherland-Lindemann ${ }^{39}$ ratio $L$ is defined to be the average rootmean-square deviation of a crystal particle from its average equilibrium position divided by the nearest neighbor distance, $d$.

We were unable to find solutions for the square lattice over a wide range of density using the Gaussian method. This indicates, as expected, that the square lattice is unstable, and that this is a positive feature of our density functional theory. For the triangular crystal, stable solutions do exist, and in Table II we present the equilibrium densities, Gaussian width $\epsilon$, imperfect crystal parameter $A$, and Sutherland-Lindemann ratio $L$, along with the results of more approximate density functional theories, ${ }^{18-21}$ which will be discussed in Sec. V.

We present results for both Gaussian and Fourier methods and, although the formalism outlined in Sec. II deals only with the perfect crystal case, we include both perfect and imperfect cases for comparison. We do not recommend the imperfect crystal calculation because it does not include any input concerning the vacancies it claims to describe. ${ }^{40} \mathrm{We}$ also include the perfect crystal Gaussian result for the Leutheusser direct correlation function. We have used 40 order parameters for the Gaussian method and 50 for the Fourier space method. The Gaussian approximation with the Baus-Colot $c(k)$ predicts a liquid coexistence density of 0.8961 , a Gaussian width of 0.08603 , and a fractional density change of 0.0588 . The Fourier method with the same $c(k)$ predicts a liquid coexistence density of 0.8784 and a fractional density change of 0.0878 . The predicted liquid coexistence densities are very close to the simulation result $\mathrm{t}^{22}$ of 0.88 . The predicted fractional density changes are somewhat larger than the value of 0.05 measured by the simulation, but considering the neglect of fluctuations inherent in any mean field theory, this is as one would expect.

One of the advantages of the Fourier method is its ability to give nonisotropic densities; hence, we show in Fig. 2 the Jacobian weighted density, $2 \pi r \rho(r)$, in the $(1,0)$ and $(1,1)$ directions for both Fourier and Gaussian solutions.

\section{FREEZING OF HARD SPHERES: HCP VS FCC CRYSTALS}

In this section, we discuss the results of our calculations (both Gaussian and Fourier) for the freezing of the hard sphere liquid into an hcp crystal and compare them to our previous results for the fcc crystal in paper I. We also compare our results to those of other researchers ${ }^{8,13,16,19,40,44,45,48}$ who

TABLE I. Values of the function $f(n)$ in Landau theory.

\begin{tabular}{lrr}
\hline$n$ & $f_{\text {bcc }}(n)$ & $f_{\text {fcc }}(n)$ \\
\hline 2 & 12 & 8 \\
3 & 48 & 0 \\
4 & 540 & 216 \\
5 & 4320 & 0 \\
6 & 42240 & 8000 \\
\hline
\end{tabular}




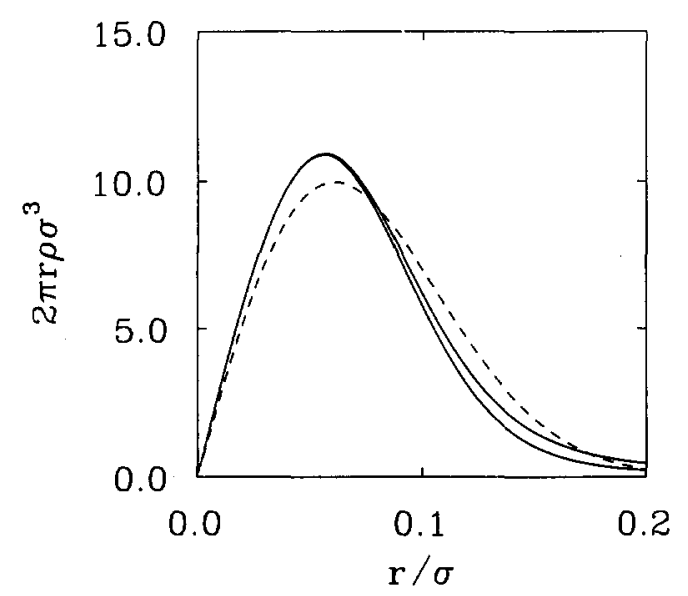

FIG. 2. The Jacobian weighted hard disk crystal density from the Gaussian approximation, which assumes an isotropic density (dashed line), and from the Fourier expansion method (solid lines) along the $(1,0)$ direction (upper solid line) and along the $(1,1)$ direction (lower solid line).

have investigated the hard sphere liquid to crystal transition with density functional theories.

The technical details of applying both the Gaussian and Fourier space methods to the hcp lattice are contained in the Appendix. A few definitions are necessary for our subsequent discussion. The hop unit cell is defined by

$$
\begin{aligned}
& \mathbf{a}_{1}=a \mathbf{i}, \\
& \mathbf{a}_{2}=-(a / 2) \mathbf{i}+(\sqrt{3} a / 2) \mathbf{j},
\end{aligned}
$$

and

$$
\mathbf{a}_{\mathbf{3}}=c \mathbf{k},
$$

where $i, j$, and $k$ are the Cartesian unit vectors in the orthogonal $x, y$, and $z$ directions, respectively. The average nearest neighbor separation is given by $d=a$; the separation of neighboring hexagonal planes, by $c / 2$. In an ideal hcp lattice, the ratio of $c$ to $a$ is $(8 / 3)^{1 / 2}$. Each unit cell of this lattice contains two particle sites displaced from the origin of the unit cell by

$$
\tau_{1}=(1 / 3) \mathbf{a}_{1}+(2 / 3) \mathbf{a}_{2}+(1 / 4) \mathbf{a}_{3},
$$

and

$$
\tau_{2}=(2 / 3) \mathbf{a}_{1}+(1 / 3) \mathbf{a}_{2}+(3 / 4) \mathbf{a}_{3} .
$$

In this work we use the analytic solution by Wertheim ${ }^{41}$ and by Thiele $^{42}$ of the approximate Percus-Yevick ${ }^{43}$ integral equation for the liquid direct correlation function,

$$
c(r)= \begin{cases}\left(1+\frac{1}{2} \xi r^{3}\right) \lambda_{1}+6 \xi r \lambda_{2}, & r<1, \\ 0, & r \geqslant 1,\end{cases}
$$

where the packing fraction, $\xi=(\pi / 6) \rho_{L}, \lambda_{1}=(1+2 \xi)^{2} /$ $(1-\xi)^{4}, \lambda_{2}=-(1+\xi / 2)^{2} /(1-\xi)^{4}$.

In the Gaussian method, a constrained minimization of the functional (2.5) is performed. For the fcc crystal, the functional is minimized with respect to the Gaussian width $\epsilon$ and the fractional density change, $\eta$. The hop lattice requires the additional minimization of the functional with respect to the distortion of the hcp $c / a$ ratio from its ideal value of $\left(\frac{8}{3}\right)^{1 / 2}$.

Our hcp results are identical to our fcc lattice results within numerical accuracy and are summarized in Table III along with fcc results of other researchers. The $c / a$ ratio for the lowest free energy crystal is found to be exactly the ideal lattice value.

We also allow the Gaussian density to have a different width parameter in the $z$ direction than in the $x$ and $y$ directions. Unlike fcc, this anisotropy is not forbidden within the hcp symmetry. The minimum value of the free energy occurs when the two width parameters are equal (that is, for a spherical Gaussian density). The equations necessary for this modification are straightforward generalizations of the Gaussian equations derived in Sec. II.

In addition to the hcp and fcc lattices, we investigated the stability of the body centered cubic (bcc) lattice. We were not

\begin{tabular}{|c|c|c|c|c|c|c|}
\hline & $\begin{array}{l}\text { Liquid } \\
\text { density }\end{array}$ & $\begin{array}{l}\text { Solid } \\
\text { density }\end{array}$ & $\begin{array}{c}\text { Gaussian } \\
\text { width }\end{array}$ & $\begin{array}{c}\text { Fractional } \\
\text { density } \\
\text { change }\end{array}$ & $\begin{array}{l}\text { Lindemann } \\
\text { ratio }\end{array}$ & $\begin{array}{l}\text { "Defect } \\
\text { density" }\end{array}$ \\
\hline \multicolumn{7}{|l|}{$\begin{array}{l}\text { Density } \\
\text { Functional theory }\end{array}$} \\
\hline 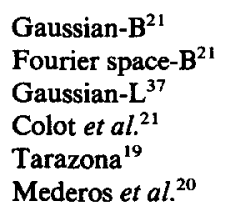 & $\begin{array}{l}0.8961 \\
0.8784 \\
0.9250 \\
0.908 \\
0.8016 \\
0.8062\end{array}$ & $\begin{array}{l}0.9488 \\
0.9555 \\
0.9839 \\
0.928 \\
0.8042 \\
0.8070\end{array}$ & $\begin{array}{c}0.08603 \\
\cdots \\
0.06062 \\
0.1302 \\
\cdots \\
\cdots\end{array}$ & $\begin{array}{l}0.0588 \\
0.0878 \\
0.0637 \\
0.0220 \\
0.0032 \\
0.0015\end{array}$ & $\begin{array}{l}0.0780 \\
0.0923 \\
0.0560 \\
0.117 \\
\cdots \\
\cdots\end{array}$ & $\begin{array}{l}\cdots \\
\cdots \\
\cdots \\
\cdots \\
\cdots \\
\cdots\end{array}$ \\
\hline \multicolumn{7}{|l|}{ Simulation } \\
\hline $\begin{array}{l}\text { Alder et al. } \\
\text { Hoover } \text { et al. }^{23}\end{array}$ & $\begin{array}{l}0.880 \\
0.878\end{array}$ & $\begin{array}{l}0.9121 \\
0.9218\end{array}$ & $\begin{array}{l}\cdots \\
\cdots\end{array}$ & $\begin{array}{l}0.0365 \\
0.0499\end{array}$ & $\begin{array}{l}\cdots \\
\cdots\end{array}$ & $\begin{array}{l}\cdots \\
\cdots\end{array}$ \\
\hline \multicolumn{7}{|l|}{ Imperfect crystal } \\
\hline $\begin{array}{l}\text { Gaussian-B } \mathbf{B}^{21} \\
\text { Fourier space- } \mathbf{B}^{21}\end{array}$ & $\begin{array}{l}0.868 \\
0.8622\end{array}$ & $\begin{array}{l}0.9000 \\
0.9077\end{array}$ & $\begin{array}{c}0.0627 \\
\cdots\end{array}$ & $\begin{array}{l}0.0369 \\
0.0528\end{array}$ & $\begin{array}{l}0.0579 \\
0.0701\end{array}$ & $\begin{array}{l}0.914 \\
0.922\end{array}$ \\
\hline
\end{tabular}
able to find any stable or metastable solutions for this lattice over a wide range of densities. This is physically correct, and an encouraging feature of density functional theory.

Based on physical intuition, one expects that both lattices,

TABLE II. Freezing of hard disks. 
hcp and fcc, will give very similar results. On the other hand, the lattice sums in the two functionals, $\Delta \beta \widetilde{\omega}_{\mathrm{fcc}}$ and $\Delta \beta \widetilde{\omega}_{\text {hcp }}$ converge in different ways and, from this point of view, it is rather surprising that numerically indistinguishable results are obtained. Figure 3 shows the value of $\Delta \beta \widetilde{\omega}$ as terms are added in the lattice sum in Eq. 2.5 plotted against the magnitude of the lattice vector of the last term added, $k_{\text {last }}$. Note that if the two summations are truncated at some wave vector before they have converged, the resulting free energies, and hence relative stabilities, would not necessarily be equal for the two lattice types.

The crystal density is periodic in nature, and hence, is represented by a discrete Fourier sum. Thus, the Fourier transform of the direct correlation function $c(k)$ is sampled at discrete magnitudes of the reciprocal lattice vectors in the lattice sums, as shown in Fig. 4. Note that the hcp sum selects different wave vector magnitudes than the fcc sum. Consequentially, errors in the input liquid structure $c(k)$ which would go unnoticed when considering one lattice type could cause serious errors in the free energy of a different lattice type. Figures 3 and 4 also show that, due to the lower symmetry of the hcp lattice, calculations of free energy for this lattice require many more sets of reciprocal lattice vectors in the lattice sum than would be necessary in the fcc case. In our calculations, 500 sets were used in the hcp case and 200 sets in the fcc case.

The justification for assuming a Gaussian form for the crystal density is the knowledge that the full (Fourier) solution is nearly Gaussian, as is the case for fcc hard spheres (Table III). Using the minimized Gaussian density as an initial guess, we have attempted to solve the Fourier method equations (2.12)-(2.14) (also see the Appendix), using the NewtonRaphson method. For a fixed bulk solid density we find a welldefined minimum in the ground potential. However, we have not completed the minimization with respect to the bulk crystal density.

At the coexisting liquid and crystal densities $\left(\rho_{L}\right.$ $=0.9836, \rho_{S}=1.1242$ ) of the fcc Fourier method calculation, the hcp Fourier method yields a (dimensionless) grand potential of -0.12 . Given the approximations in our theory, we

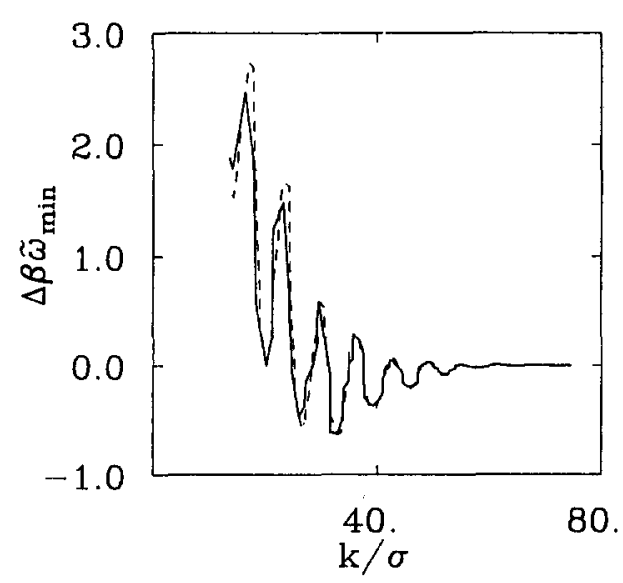

FIG. 3. The value of the grand thermodynamic potential difference $\Delta \beta \omega$ as more terms are added to the lattice sum, for both the foc lattice (solid line) and the hcp lattice (dashed line). Note that the fully converged results at the extreme right-hand side of the figure contain 200 and $500 \mathrm{RLVs}$, respectively.

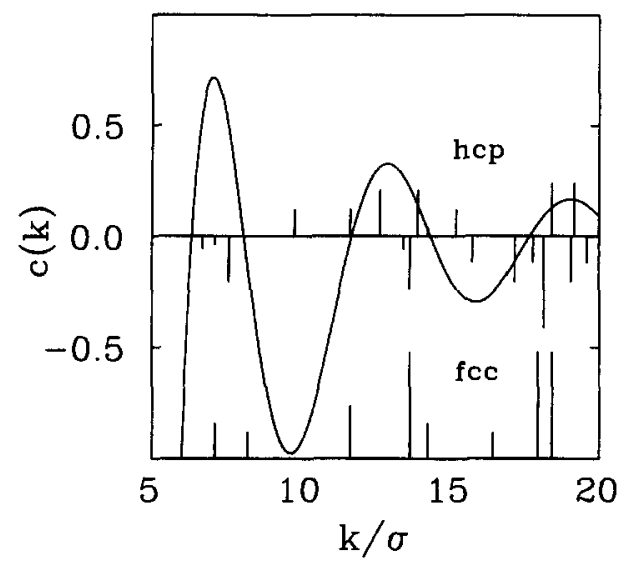

FIG. 4. The Fourier transform of the hard sphere liquid direct correlation function near freezing $\left(\rho_{L}=0.985\right)$. The vertical lines show the magnitudes of the foc and hcp reciprocal lattice vectors (RLVs). The length of the lines is proportional to the weighting factor $n_{i} f_{i}$, where $n_{i}$ is the degeneracy of the RLV symmetry set " $i$ " and $f_{i}$ is the corresponding geometric structure factor defined in the Appendix (A 2). Note that $f_{i}=1$ for all fcc RLVs, and that $f_{i}$ for the hep vectors can be negative.

conclude that the two close-packed structures, of hard spheres $\mathrm{fcc}$ and hcp, have approximately the same stability.

There have been a number of predictions of the hard sphere liquid to crystal transition by other researchers who have produced a variety of results as seen in Table III. It is of interest to see how variations on the techniques used in this paper change the predicted coexistence point. We have restricted the entries in the table to results which seem well converged; for instance, we have avoided early papers where the Fourier series was truncated at a small number of order parameters. Notice that the bulk of these entries employ the Gaussian approximation which, although lacking in generality, is much easier to apply.

The work of McCoy, Singer, and Chandler ${ }^{13}$ is most similar to our own differing only in that they do not include the $c^{(3)}(0,0)$ term. Following the Fourier space methodology of paper I, they found coexistence in the fcc lattice and, by using the Gaussian method, found coexistence in both the fcc and hcp lattices. Again, fcc and hep crystals are found to have virtually identical stability. Jones and Mohanty ${ }^{40}$ had previously applied the Gaussian method to the imperfect crystal fcc and bcc lattices [also without the $c^{(3)}(0,0)$ term]. As in our calculation, they failed to find a solution corresponding to a bcc solid. Notice that omission of the $c^{(3)}(0,0)$ term lowers the liquid density and increases the fractional density change to nearly twice the experimental value.

In addition to omitting the $c^{(3)}(0,0)$ term, Baus and Co$\operatorname{lot}^{17}$ make use of a hypothetical reference liquid, with a uniform density intermediate between the liquid and crystal density. This reference density is chosen arbitrarily by requiring that the first peak of its $c(k)$ coincide with the smallest reciprocal lattice vector of the crystal lattice. The resulting density is much higher than equilibrium liquid densities. The corresponding direct correlation function is found by extrapolating conventional liquid state theory to these higher densities. This procedure gives adequate predictions for the coexistence properties, but it is not clear how the method can be applied to 
TABLE III. Freezing of hard spheres

\begin{tabular}{|c|c|c|c|c|c|c|}
\hline & $\begin{array}{l}\text { Liquid } \\
\text { density }\end{array}$ & $\begin{array}{l}\text { Solid } \\
\text { density }\end{array}$ & $\begin{array}{l}\text { Gaussian } \\
\text { width }\end{array}$ & $\begin{array}{c}\text { Fractional } \\
\text { density } \\
\text { change }\end{array}$ & $\begin{array}{l}\text { Lindemann } \\
\text { ratio }\end{array}$ & $\begin{array}{l}\text { Defect } \\
\text { density }\end{array}$ \\
\hline \multicolumn{7}{|l|}{$\begin{array}{l}\text { Density } \\
\text { Functional theory }\end{array}$} \\
\hline $\begin{array}{l}\text { Laird et al. }(\mathrm{F})^{8} \\
\text { Laird } \text { et al. }(\mathrm{G})^{8} \\
\text { McCoy } \text { et al. }(\mathrm{G})^{13} \\
\text { McCoy } \text { et al. }(\mathrm{F})^{13} \\
\text { Igloi } \text { et al. }(\mathrm{F})^{16} \\
\text { Tarazona }(\mathrm{G})^{19} \\
\text { Baus et al. }(\mathrm{G})^{45} \\
\text { Curtin } \text { et al. }(\mathrm{G})^{47}\end{array}$ & $\begin{array}{l}0.9836 \\
0.9850 \\
0.9676 \\
0.965 \\
0.975 \\
0.9433 \\
0.993 \\
0.892\end{array}$ & $\begin{array}{l}1.1241 \\
1.1258 \\
1.150 \\
1.148 \\
1.076 \\
1.0609 \\
1.083 \\
1.025\end{array}$ & $\begin{array}{l}\cdots \\
0.0520 \\
0.04508 \\
\cdots \\
\cdots \\
\approx .13 \\
0.066 \\
0.0598\end{array}$ & $\begin{array}{l}0.1429 \\
0.1430 \\
0.189 \\
0.190 \\
0.104 \\
0.125 \\
0.091 \\
0.149\end{array}$ & $\begin{array}{l}0.065 \\
0.059 \\
0.052 \\
\cdots \\
0.070 \\
\approx .14 \\
0.074 \\
0.104\end{array}$ & $\begin{array}{l}\cdots \\
\cdots \\
\cdots \\
\cdots \\
\cdots \\
\cdots \\
\cdots \\
\cdots\end{array}$ \\
\hline \multicolumn{7}{|l|}{ Simulation } \\
\hline $\begin{array}{l}\text { Hoover et al. }{ }^{44} \\
\text { Imperfect crystal }\end{array}$ & $0.94-0.96$ & $1.04-1.05$ & $\cdots$ & $0.08-0.11$ & 0.14 & $\cdots$ \\
\hline $\begin{array}{l}\text { McCoy et al. }(\mathrm{F})^{13} \\
\text { Jones et al. }(\mathrm{G})^{40}\end{array}$ & $\begin{array}{l}0.944 \\
0.9461\end{array}$ & $\begin{array}{l}1.0501 \\
1.0525\end{array}$ & $\begin{array}{c}\cdots \\
0.0415\end{array}$ & $\begin{array}{l}0.112 \\
0.112\end{array}$ & $\begin{array}{c}\ldots \\
0.0477\end{array}$ & $\begin{array}{l}0.897 \\
0.898\end{array}$ \\
\hline
\end{tabular}

systems which do not have accurate liquid state theories that can be extrapolated to such high densities. This method predicts correctly that the square lattice is unstable. Crystal-like solutions for the bec symmetry were found only at unphysically high densities. However, the fcc lattice was found to have coexistence properties identical to the ones found for the hcp lattice, and the preferred hcp lattice was found to be ideal.

Igloi and Hafner ${ }^{16}$ expand the free energy functional in a Taylor series about a hypothetical reference liquid rather than about the equilibrium liquid. Their reference density is chosen to give the lowest liquid coexistence density. They claim that this choice reduces the effect of higher order terms in the Taylor series. The most recent results of Igloi ${ }^{16}$ on both the fcc and hcp lattices using a Fourier space method show no significant difference between the two lattices. It will be interesting to test this method using the correct $k=0$, perfect crystal Fourier space method.

Finally, Tarazona ${ }^{19}$ has introduced a method, related to methods used in the theory of the inhomogeneous electron gas, which incorporates higher order terms in the density expansion. Curtin, Ashcroft, and Runge ${ }^{46,47,48}$ have refined this theory. Here the liquid and solid chemical potentials are not required to be equal at all points in space. Instead, only the spatially averaged chemical potentials are equal. It is interesting that their coexistence densities tend to be lower than the simulation results, while the previously discussed theories tend to give densities which are higher than simulation. Another difference is that the direct correlation function used in a Tarazona type theory is that of a very low density liquid. As discussed by the authors, a surprising feature of this theory is that it predicts a stable hard sphere bcc crystal, which is known never to be stable.

In summary, we believe that density functional theory can predict accurately the freezing transition in a simple liquid, and that the version presented in paper I and developed here is consistent and well founded.

\section{CONCLUSIONS}

In this paper we have shown that the density functional theory developed in paper I can be used to predict the freezing of hard particles in two and three dimensions. In contrast, traditional Landau theory does not predict the quantitative or qualitative features of the transition.

The implementation of the full, Fourier space freezing theory for crystals with a basis of more than one atom is more intricate, but as shown in the Appendix, it may be accomplished by using standard crystallographic methods. We have established a technique for comparing the relative stability of different crystal structures, and further applications are underway.

\section{ACKNOWLEDGMENTS}

This research was supported by the (U.S.) National Science Foundation and the Ford Motor Company Fund under the PYI program. We thank Dr. Shep Smithline for many helpful discussions.

\section{APPENDIX}

Performing the Fourier analysis to minimize Eq. (2.1) is relatively straightforward for systems with one particle per Bravias lattice site (such as fcc, bcc, simple hexagonal, etc.). When there is more than one particle associated with a lattice site the procedure becomes more complicated. For such systems, the Fourier expansion of the solid density can be written

$$
\rho(\mathbf{r})=\rho_{L}\left[1+\eta+\sum_{\mathbf{k}_{n} \neq \mathbf{0}} \mu_{\mathbf{k}_{n}} f_{\mathbf{k}_{n}} e^{i \mathbf{k}_{n} \cdot \mathbf{r}}\right],
$$

where the atomic structure factors $\mu_{\mathrm{k}}$ are determined by the shape of the density peak about each particle site and the geometric structure factors $f_{\mathbf{k}}$ are determined by the $n_{\tau}$ atomic basis vectors $\tau_{i}\left(i=1, n_{\tau}\right)$ associated with each Bravias lattice site: 


$$
f_{\mathbf{k}_{n}}=\frac{1}{n_{\tau}} \sum_{i=1}^{n_{\tau}} e^{i k_{n} \cdot \tau_{i}}
$$

Two reciprocal lattice vectors (RLVs) $k_{n}$ and $k_{m}$ are said to be in the same symmetry set if their corresponding atomic structure factors are required to be equal by symmetry. All vectors $\mathbf{k}_{n}$ in a symmetry set have the same magnitude. If we order the symmetry sets by magnitude (some sets will have the same magnitude, in which case the order is arbitrary) and index them by the integer $i$, we can write the expansion (A1) as

$$
\rho(\mathbf{r})=\rho_{L}\left[1+\eta+\sum_{i \neq 0} \mu_{i} f_{i} \Gamma_{i}(\mathbf{r})\right],
$$

where $\mu_{i}$ is the value of the atomic structure factor shared by the members of the set $i$ and $f_{i}$ is the value of the geometric structure factor for a specific RLV $\mathbf{k}_{i}$ chosen to represent the set $i$, and

$$
\Gamma_{i}(\mathbf{r})=\sum_{\mathbf{k}, j} \frac{f_{\mathbf{k}_{j}}}{f_{i}} e^{\boldsymbol{a k}_{\mathbf{k}} \cdot \mathbf{r}} .
$$

Using expansion (A4) together with the fact that the geometric structure factors for two RLVs in the same symmetry set can differ only by a phase factor, the Gaussian method expression for the functional (2.1) becomes

$$
\begin{aligned}
\Delta \beta \widetilde{\omega}=1 & -(1+\eta)\left[\frac{5}{2}+\ln \rho_{L}+\frac{3}{2} \ln \left(\pi \epsilon^{2}\right)\right] \\
& -\frac{1}{2} \eta^{2} c(0)-\frac{1}{2} \sum_{i \neq 0} n_{i} c\left(\left|\mathbf{k}_{i}\right|\right) f_{i}^{2} \mu_{i}^{2} \\
& -\frac{1}{6} \eta^{3} c^{(3)}(0,0),
\end{aligned}
$$

where $n_{i}$ is the number of RLVs in set $i$. The Fourier method equations (2.12) become

$$
\begin{aligned}
\mu_{i}+\delta_{i, 0}= & \frac{1}{n_{i} f_{i} \Delta} \int_{\Delta} d \mathbf{r} \Gamma_{i}(\mathbf{r}) \exp \{\lambda+c(0) \eta \\
& \left.+\frac{1}{2} c^{(3)}(0,0) \eta^{2}+\sum_{j \neq 0} c\left(\left|\mathbf{k}_{j}\right|\right) \mu_{j} f_{j} \Gamma_{j}(\mathbf{r})\right\},
\end{aligned}
$$

where the equations for each member of the symmetry set $i$ have been combined into one equation for $\mu_{i}$.

The hcp lattice is a simple example of crystal structure with a multiparticle atomic basis. The hcp unit cell is defined by the real space vectors $a_{1}, a_{2}$, and $a_{3}$ defined in Eq. (5.1). The volume of the unit cell $\Delta_{\mathrm{hcp}}$ is given by

$$
\Delta_{\mathrm{hcp}}=\frac{\sqrt{3}}{2} a^{2} c .
$$

The ideal hep crystal has a $c / a$ ratio equal to $(8 / 3)^{1 / 2}$ although, in general, this value is not constrained by symmetry. The atomic basis set contains two vectors:

$$
\tau_{1}=\left(\frac{1}{3},-\frac{1}{3}, \frac{1}{4}\right)
$$

and

$$
\tau_{2}=\left(-\frac{1}{3}, \frac{1}{3},-\frac{1}{4}\right),
$$

where the fractional coordinate $(x, y, z)=x \mathbf{a}_{1}+y \mathbf{a}_{2}+z \mathbf{a}_{3}$.

The reciprocal lattice vectors are

$$
(h k l)=h \mathbf{a}_{1}^{*}+k \mathbf{a}_{2}^{*}+l \mathbf{a}_{3}^{*}
$$

with

$$
\begin{aligned}
& \mathbf{a}_{1}^{*}=\frac{2 \pi}{a}\left(\mathbf{i}+\frac{\sqrt{3}}{3} \mathbf{j}\right), \\
& \mathbf{a}_{2}^{*}=\frac{4 \pi \sqrt{3}}{3 a} \mathbf{j},
\end{aligned}
$$

and

$$
\mathbf{a}_{3}^{*}=\frac{2 \pi}{c} \mathbf{k},
$$

where $\mathbf{i}, \mathbf{j}$, and $\mathbf{k}$ are the standard Cartesian unit vectors. The magnitude of the $\mathrm{RLV}(h k l)$ is given by

$$
\left|\mathbf{k}_{h k l}\right|=\frac{2 \pi}{a}\left[\frac{4}{3}\left(h^{2}+h k+k^{2}\right)+\left(\frac{a}{c} l\right)^{2}\right]^{1 / 2} .
$$

Equation (A2), together with the atomic basis (A8), gives the following expression for the geometric structure factor for $\operatorname{RLV}(h k l)$ :

$$
f_{h k l}=\cos \left\{\pi\left[\frac{2(h-k)}{3}+\frac{l}{2}\right]\right\} .
$$

Note that the geometric structure factors vanish for RLVs with $h-k=0(\bmod 3)$ and $l$ odd. These vectors are called systematic absences and do not contribute to the Fourier sums.

There are three real space symmetry elements that determine the hop reciprocal space symmetry sets. The first is a center of inversion at the origin:

$$
\rho(x, y, z)=\rho(-x,-y,-z) .
$$

The effect of this symmetery on the reciprocal space is that the atomic and geometric structure factors associated with $R L V$ $(h k l)$ are equal to the corresponding quantities for the vector $(\bar{h}, \bar{k}, \bar{l})$ (the symbol $\bar{h}$ is standard crystallographic notation for "minus" h). Since in general

$$
\mu_{\mathrm{k}} f_{\mathrm{k}}=\mu_{-\mathrm{k}}^{*} f_{-\mathrm{k}}^{*},
$$

where the ${ }^{*}$ denotes complex conjugation, the center of inversion at the origin guarantees that the structure factors are real.

The center of inversion involves only a simple point group operation (inversion). The other two elements involve compound symmetry operations that are combinations of point group operations and spatial translations. To see how such symmetries of the real space crystal affect the structure factors consider a hypothetical compound operation $S=T P$, where $P$ is a point group operation (rotation, inversion, reflection or improper rotation) and $T$ represents a translation by a vector $t$. The real space symmetery $S$ implies a relation between the structure factors corresponding to RLV $(h k l)$ and the RLV formed by applying the inverse of $P$ to the vector $(h k l), P^{-1}$ $(h k l)$. The atomic structure factors for the two vectors are equal;

$$
\mu_{(h k l)}=\mu_{P^{-1}(h k l)} .
$$

The geometric structure factors for RLVs $(h k l)$ and $P^{-1}(h k l)$ differ by a phase factor determined by the translational component of $S$ : 


$$
f_{(h k l)}=f_{P^{-1}(h k l)} e^{i k_{h k l^{t}}} \text {. }
$$

The two other symmetery operations needed to define the symmetry sets are (1) a sixfold screw axis corresponding to a rotation by $\pi / 3$ radians about the $z$ axis followed by a translation by half a unit cell in the $z$ direction:

$$
\rho(x, y, z)=\rho(x-y, x, z+1 / 2),
$$

and (2) a glide plane corresponding to a reflection across the plane $x=y$ also followed by a translation by half a unit cell in the $z$ direction:

$$
\rho(x, y, z)=\rho(y, x, z+1 / 2) .
$$

Using relation (A16), the sixfold screw axis implies that

$$
\begin{aligned}
f_{h k l} & =f_{(\bar{k}, h+k, l)} e^{i \pi l}, \\
& =f_{(\bar{k}, h+k, l)}(-1)^{l} .
\end{aligned}
$$

Similarly, the presence of the glide plane implies that

$$
f_{(h k l)}=f_{(k h l)}(-1)^{l} \text {. }
$$

It is important to note that another choice of origin besides the one used to define the atomic basis vectors (A8) would unnecessarily complicate the relations between the structure factors in a symmetry set due to the lack of one or more of the above symmetry elements.

As a consequence of the above symmetries, the RLVs ( $h k l$ ) such that $0 \leqslant h \leqslant k, k \geqslant 1$ and $l \geqslant 0$ each represent a unique symmetry related set of vectors. The entire reciprocal lattice can be derived from this set by application of the above three symmetery operations. Ordered by magnitude these vectors represent the sets summed over in Eq. (A3).

Because of the symmetry of the crystal, the integrations contained in Eq. (A6) do not have to be performed over the entire unit cell. A region of minimum volume which can be integrated over to yield correct results is called an asymmetric unit. For hcp, the asymmetric unit is the volume defined by the triangular prism with verticies: $(0,0,0),(1 / 3,2 / 3,0)$, $(2 / 3,1 / 3,0),(0,0,1 / 4),(1 / 3,2 / 3,1 / 4)$, and $(2 / 3,1 / 3,1 / 4)$. The volume of this region is $1 / 24$ th of the volume of the unit cell.

${ }^{1}$ J. L. Lebowitz and J. K. Percus, J. Math. Phys. 4, 116 (1963).

${ }^{2}$ F. H. Stillinger and F. P. Buff, J. Chem. Phys. 37, 1 (1962).

${ }^{3}$ D. Mermin, Phys. Rev. A 137, 1441 (1965).

${ }^{4}$ R. Evans, Adv. Phys. 28, 143 (1979).

${ }^{5}$ T. V. Ramakrishnan and M. Yussouff, Phys. Rev. B 19, 2775 (1979).
${ }^{6}$ A. D. J. Haymet and D. W. Oxtoby, J. Chem. Phys. 84, 1769 (1986).

${ }^{7}$ A. D. J. Haymet, J. Chem. Phys. 78, 4641 (1983).

${ }^{8}$ B. B. Laird, J. D. McCoy, and A. D. J. Haymet, J. Chem. Phys. 87, 5449 (1987).

${ }^{9}$ A. D. J. Haymet, Annu. Rev. Phys. Chem. 38, 89 (1987).

${ }^{10} \mathrm{~J}$. L. Barrat, M. Baus, and J. P. Hansen, Phys. Rev. Lett. 56, 1063 (1986).

${ }^{11}$ S. J. Smithline and A. D. J. Haymet, J. Chem. Phys. 86, 6486 (1987).

12S. J. Smithline, S. W. Rick, and A. D. J. Haymet, J. Chem. Phys. 88, 2004 (1988).

${ }^{13}$ J. D. McCoy, S. Singer, and D. Chandler, J. Chem. Phys. 87, 4853 (1987).

${ }^{14}$ K. Ding, D. Chandler, S. J. Smithline, and A. D. J. Haymet, Phys. Rev. Lett. 59, 1698 (1987).

${ }^{15}$ M. Yussouff, Phys. Rev. B 23, 5871 (1981).

${ }^{16}$ F. Igloi, J. Phys. C 19, 6907 (1986); see also, F. Igloi and J. Hafner, 19, 5799 (1986).

${ }^{17}$ M. Baus and J. L. Colot, Mol. Phys. 55, 653 (1985); see also 57, 809 (1986).

${ }^{18}$ T. V. Ramakrishnan, Phys. Rev. Lett. 48, 541 (1982).

${ }^{19}$ P. Tarazona, Phys. Rev. A 31, 2672 (1985); 32,3148 (E) (1985); Mol. Phys. 52, 81 (1984).

${ }^{20}$ L. Mederos, P. Tarazona, and G. Navascues, Phys. Rev. B 35, 3376 (1986).

${ }^{21}$ J. Colot and M. Baus, Phys. Lett. A 119, 135 (1986).

${ }^{22}$ B. Alder and T. Wainwright, Phys. Rev. 127, 359 (1962).

${ }^{23}$ W. Hoover and F. Ree, J. Chem. Phys. 49, 3609 (1968).

${ }^{24}$ A. D. J. Haymet and D. W. Oxtoby, J. Chem. Phys. 84, 1769 (1986).

${ }^{25}$ R. L. Jacobs, J. Phys. C 16, 273 (1983).

${ }^{26}$ L. D. Landau, in Collected Papers of L. D. Landau, edited by D. ter Haar (Gordon and Breach, New York, 1965), p. 193.

${ }^{27}$ K. G. Wilson and J. Kogut, Phys. Reports 12, 75 (1974).

${ }^{28}$ M. N. Barber, Phys. Reports 29, 1 (1977).

${ }^{29}$ S. Alexander and J. P. McTague, J. Phys. Rev. Lett. 41, 702 (1978); see also J. C. and P. Tolédano, The Landau Theory of Phase Transitions, (World Scientific, Singapore, 1987).

${ }^{30}$ P. Bak, Phys. Rev. Lett. 54, 1517 (1985).

${ }^{31}$ D. Mermin and S. Troie, Phys. Rev. Lett. 54, 1524 (1985).

${ }^{32}$ W. Klein and F. Leyvraz, Phys. Rev. Lett. 57, 2845 (1986).

${ }^{33}$ W. H. Shih, Z. Q. Wang, X. C. Zeng, and D. Stroud, Phys. Rev. A 35, 2611 (1987); see also, S. M. Moore and H. J. Raveche, J. Chem. Phys. 85, 6039 (1986).

${ }^{34}$ A. D. J. Haymet and D. W. Oxtoby, J. Chem. Phys. 74, 2559 (1981).

${ }^{35}$ D. W. Oxtoby and A. D. J. Haymet, J. Chem. Phys. 76, 6262 (1982); see also, B. B. Laird and A. D. J. Haymet, in Proceedings of the Materials Research Society (Materials Research Society, Pittsburgh, PA, 1986), p. 67.

${ }^{36}$ A. D. J. Haymet, Phys. Rev. Lett. 52, 1013 (1984).

${ }^{37}$ E. Leutheusser, J. Chem. Phys. 84, 1050 (1986).

${ }^{38}$ F. Lado, J. Chem. Phys. 49, 3092 (1968).

${ }^{39}$ W. Sutherland, Philos. Mag. 30, 318 (1890); F. A. Lindemann, Phys, Z. 11, 609 (1910).

${ }^{40} \mathrm{G}$. Jones and U. Mohanty, Mol. Phys. 54, 1241 (1985).

${ }^{4}$ 'M. S. Wertheim, Phys. Rev. Lett. 10, 321 (1963).

${ }^{42}$ E. Thiel, J. Chem. Phys. 39, 474 (1963).

${ }^{43}$ J. K. Percus and G. J. Yevick, Phys. Rev. 110, 1 (1958).

${ }^{44}$ W. Hoover and B. Alder, J. Chem. Phys. 46, 686 (1967); W. Hoover and F. Ree, ibid. 47, 4873 (1967); W. Hoover and B. Alder, ibid. 60, 1254 (1974).

${ }^{45}$ M. Baus and J. Colot, Mol. Phys. 55, 653 (1985); J. Colot and M. Baus, ibid. 56, 807 (1985)

${ }^{46}$ W. A. Curtin and N. W. Asheroft, Phys. Rev. Lett. 56, 2775 (1986); 57, 1192 (E) (1986).

${ }^{47}$ W. Curtin and K. Runge, Phys. Rev. A 35, 4755 (1987)

${ }^{48}$ W. A. Curtin and N. W. Ashcroft, Phys. Rev. A 32, 2909 (1985). 